\title{
Controlled Array of Gold Nanoparticles by Combination of Nano Imprint and Self-assembly
}

\author{
Hiroki Yamamoto ${ }^{a^{*}}$, Akira Ohnuma ${ }^{b, c}$, Bunsho Ohtani $^{\mathrm{b}}$, and Takahiro Kozawa \\ ${ }^{a}$ The Institute of Scientific and Industrial Research, Osaka University, \\ 8-1 Mihogaoka, Ibaraki, Osaka 567-0047, Japan \\ ${ }^{b}$ Institute for Catalysis, Hokkaido University, Sapporo 001-0021, Japan \\ ${ }^{c}$ Institute for the Advancement of Higher Education, Hokkaido University, Sapporo 060-0817, Japan \\ *E-mail address: hiroki@sanken.osaka-u.ac.jp
}

\begin{abstract}
The fundamental limitations associated with top-down lithographic techniques gradually have approached. One of methods to overcome these limitations is the combination technique of top-down nanofabrication such as nano imprint and bottom-up nanofabrication such as self-assembly. Nanoparticles has been expected to use as the building blocks. In order to realize this method, it is desirable to place nanometer sized components such as nanoparticles in exact positions for nano-fabrication with high precision and reproducibility. In particular, gold $(\mathrm{Au})$ nanoparticles, which covalently linked to biomolecules such as DNA, peptides, nucleic acids, proteins, and cell have attracted much attention for biosensing, bioimaging, and plasmonics application, and so on. In this study, we present a simple method of controlled array of $\mathrm{Au}$ nanoparticles on a nanopattern by the combination of self-assembly techniques bottom-up nano-fabrication such as self-assembled monolayers and top-down nano-fabrication such as nano imprint. We believe this method will contribute to studies on the observation of single biomolecules such as DNA, peptides, nucleic acids, proteins, plasmonics and so on.
\end{abstract}

Keywords: Nanoimprint, Au nanoparticles, self-assembly, self-assembled monolayers (SAMs), nanofabrication

\section{Introduction}

Nanolithography technologies less than the $100 \mathrm{~nm}$ node scale has been widely used, including the $193 \mathrm{~nm}$ immersion, electron beam (EB) [1,2], extreme ultraviolet (EUV) and nano imprint methods [3,4] and focused ion beam (FIB) lithography [5] and so on. Among these technologies, nano imprint lithography is one of the promising lithographic technologies for advanced devices because it requires no expensive equipment. Nano imprint lithography have the potential ability to fabricate less than $10 \mathrm{~nm}$ feature size. However, fundamental limitations associated with top-down lithographic techniques gradually have approached. Therefore, a new concept for scalable technologies is required to be developed and different novel techniques for nanolithography have been investigated $[6,7]$.
Recently, a new nanofabrication technique uses nanoparticles as the building blocks. In particular, it is desirable to place nanometer sized components such as nanoparticles in exact positions for nanofabrication with high precision and reproducibility. One of the promising methods for constructing such ordered structures is to chemically attach nanoparticles onto a highly ordered layer of functional molecules such as self-assembled monolayers (SAMs), which are formed by the spontaneous organization of thiolated molecules on a desired surface such as a metal, semiconductor, or insulator [8].

Up to now, a variety of techniques such as EB lithography [1], micro contact printing [9], scanning tunneling microscope lithography [10], dip-pen nanolithography [11], electrochemical etching and electrodeposition [12] have been used 
to control the arrays of nanoparticle on substrates with dimensions in the sub-100 $\mathrm{nm}$. Also, progress has been made in the assembly of metal nanoparticles for the fabrication of nanoscale structures [13], and the EB patterning of nanoparticle monolayers has been demonstrated [14]. However, it is still difficult to precisely control the attachment of nanoparticles. Therefore, it is still fascinating to develop new preparation methods of nanoparticle arrays and improve these technologies.

In previous study, we succeeded in a novel and simple method of fabricating controlled arrays of $\mathrm{Au}$ nanoparticles on a nanoscale reaction field by combining EB lithography and self-assembly techniques [15-17]. Also, we succeeded in controlled arrangement of Au nanoparticles capped with protecting ligand on $\mathrm{Au}$ nanopatterns [18] Furthermore, we succeessfully controlled the position of $\mathrm{Au}$ nanoparticles on a nanoscale reaction field by using self-assembly techniques [19]. In this study, controlled array of $\mathrm{Au}$ nanoparticles was successfully performed by the combination of top-down nanofabrication such as nano imprint and bottom-up nanofabrication such as self-assembly.

\section{Experimental}

PAK-01 is used as a resin for UV imprinting of the radical polymerization type based on acrylic resin and is widely used in UV nanoimprint process [20]. PAK-01 films were spin-coated on Si substrates. The imprinting was performed using an imprint equipment made by Litho Tech Japan Corporation (LTNIP-5000). They were pressed with a quartz glass mold while exposed to ultraviolet light for curing to perform UV imprinting. A quartz glass mold with various patterns including line and space, squares, isolated lines and dots, which is supplied by Toppan Printing Co. Ltd., was used in this experiment. The size of the quartz glass mold is 1 inch square. The pressing pressure and main exposure were 1.55 $\mathrm{MPa}$ and $1500 \mathrm{~mJ} / \mathrm{cm}^{2}$, respectively. Then, the PAK-01 films were treated with oxygen reactive ion etching (RIE) process in order to partially denude Si surface and form PAK-01 patterns on the Si substrate. After selective removal of bottom layer, the remaining PAK-01 pattern can be obtained. The resulting patterns was recorded using field emission-scanning electron microscopy (FE-SEM, Hitachi-hitec S-5500).

Using these remaining PAK-01 patterns, $\mathrm{Au}$ nano-patterns were fabricated on a $\mathrm{Si}$ substrate using lift-off process. The $15 \mathrm{~nm}$ thick Au layers on Ti layers was deposited by EB deposition. After EB deposition, the remained polymer film was dissolved by dipping PAK-01 into a distilled water for a few hours at $23{ }^{\circ} \mathrm{C}$.

In self-assembly procedure, Au nano-patterns is exposed to a solution of dithiol linker compound in water. Au patterns were covered with SAMs by the immersion of the Au patterned substrate into water solutions of dithiol. We selected water-soluble SAM such as poly(ethylene glycol) dithiol because our purpose is biosensing such as single-molecular observation and plasmonics application. Finally, resulting SAMs patterns were immersed into the aqueous colloidal solution of citrate-stabilized $\mathrm{Au}$ nanoparticles in order to attach the nanoparticles onto them. In the sample preparation for $\mathrm{Au}$ nanoparticles, an aqueous colloidal solution of citrate-stabilized gold nanoparticles was prepared following the method of Frens [21-23]. The structure obtained was imaged with a FE-SEM and the position control of $\mathrm{Au}$ nanoparticles was characterized.

\section{Results and Discussion}

(a)

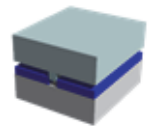

(b)

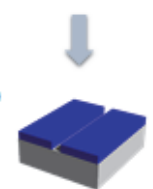

(c)

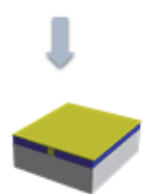

(f)

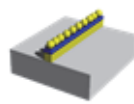

(e)

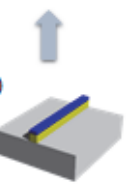

(d)

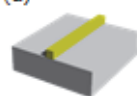

Fig. 1. Schematic process flow of our fabrication proce dure of controlled array of Au nanoparticles using the combination of nano imprint and selfassembly.

Figure 1 shows a schematic process flow of our fabrication procedure of controlled array of $\mathrm{Au}$ nanoparticles using top-down nanofabrication such as nano imprint and bottom-up nanofabrication such as self-assembly. A preliminary experiment was carried out using PAK-01. At first, varios patterns were fabricated by nano imprint lithography (step1). Secondarily, PAK films were 
treated with $\mathrm{O}_{2}$ RIE process in order to partially denude $\mathrm{Si}$ surface to form PAK-01 patterns on $\mathrm{Si}$ substrate (step2). Then, Ti layers on $\mathrm{Si}$ substrate and $\mathrm{Au}$ layers on Ti layers is deposited by $\mathrm{EB}$ deposition (step3). After EB deposition, the remained polymer film of the PAK-01 was dissolved by dipping the sample into a distilled water for a few hours at $23{ }^{\circ} \mathrm{C}$ (step4). Next, Au patterns were covered with SAMs by the immersion of the $\mathrm{Au}$ patterned substrate into aqueous solutions of dithiol (step5). Finally, SAMs patterns were immersed into the aqueous colloidal solution of citrate-stabilized $\mathrm{Au}$ nanoparticles in order to attach the nanoparticles onto them (step6).

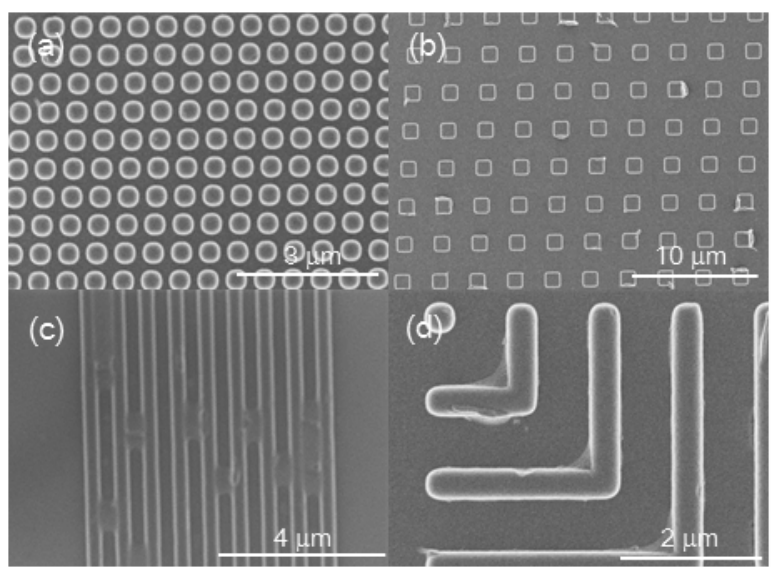

Fig. 2. SEM micrographs of micrograph of PAK-01 imprinted using quartz glass mold including (a) dots, (b) square pillars, (c) line and space pattern, and (d) elbow patterns, respectively.

Figure 2 shows SEM micrographs of PAK-01 imprinted using quartz glass mold including (a) dots, (b) square pillars, (c) line and space pattern, and (d) elbow patterns, respectively. The photo-reaction initiator is decomposed upon UV exposure and PAK-01 generates free radicals. These free radicals induce acrylic polymerization and the pattern transferred from quartz glass mold could be obtained as shown in Figure 2. Using the optimized process conditions, we succeessfully could perform transfer experiments involving 300 $\mathrm{nm}$ line and space pattern and $400 \mathrm{~nm}$ dots pattern.

Figure 3 shows a SEM micrograph of (a) the square pattern and (b) the line and space pattern imprinted using PAK-01 after $\mathrm{O}_{2}$ RIE. Although the SEM micrograph is not shown here, minimum feature sizes of $200 \mathrm{~nm}$ and other shape of patterns could be achieved. By using the patterned PAK-01 mask, $\mathrm{Au}$ layers in defined areas on the Si surface was deposited by EB deposition. Then, resulting $\mathrm{Au}$ nanopattern including dots and lines after the
$\mathrm{Au}$ deposition and the lift-off process was covered with the SAMs by standard immersion procedure. A SAM such as poly(ethylene glycol) dithiol were used in order to attach $\mathrm{Au}$ nanoparticles on $\mathrm{Au}$ nanopatterns as previous reported [15-19]. The resulting patterned SAM is used as a binding layer for the spatially selective assembly of $\mathrm{Au}$ nanoparticles.

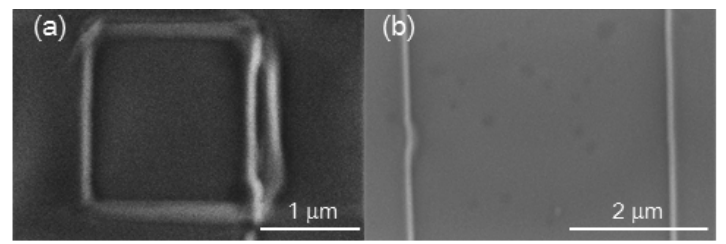

Fig. 3. SEM micrographs of (a) the square pattern and (b) the line and space pattern imprinted using PAK-01 after $\mathrm{O}_{2}$ RIE.

An aqueous colloidal solution of citrate-stabilized $\mathrm{Au}$ nanoparticles was prepared and used. Figure 4 shows a SEM micrograph of Au nanoparticles used in this study. Au nanoparticles have a diameter between 10 and $45 \mathrm{~nm}$, with an average diameter of $28 \mathrm{~nm}$. The size and shape of nanoparticles can be easily controlled by changing synthesis and process conditions in this method. Using synthesized Au nanoparticles, the controlled array of Au nanoparticles on Au nanopatterns was characterized by FE-SEM. Figure 5 (a) $\sim$ (d) show SEM micrographs of controlled array of $\mathrm{Au}$ nanoparticles supported by dithiol SAMs on $\mathrm{Au}$ patterns, which is corresponding to step 6 in Figure 1. Au nanoparticles were anchored onto Au nano pattern as shown in Figure 5. We succeeded in controlled array of $\mathrm{Au}$ nanoparticles on the resulting dithiol SAM patterns.

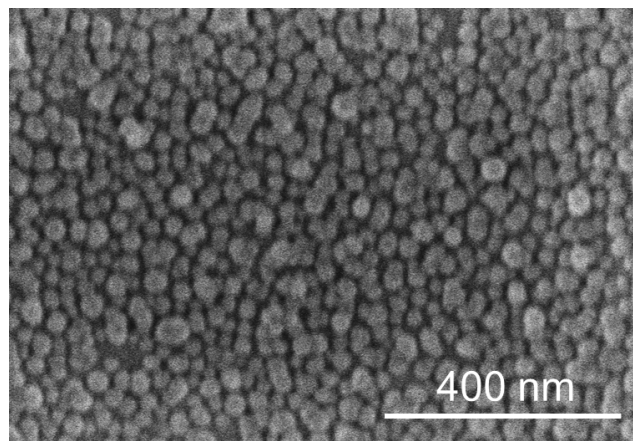

Fig. 4. A SEM micrograph of $\mathrm{Au}$ nanoparticles. They have a diameter between 10 and $45 \mathrm{~nm}$, with an average diameter of $28 \mathrm{~nm}$.

In this study, the position control of $\mathrm{Au}$ nanoparticles has been successfully fabricated by the combination technique of top-down nanofabrication such as nano imprint and 
bottom-up nanofabrication such as self-assembly. The diameter and the pitch of Au nanodots and line and pitch of line and space patterns can be controlled by using imprinted PAK-01 mask. This method opens the door to various applications in electronics, optics, biosensing, bioimaging and so on.

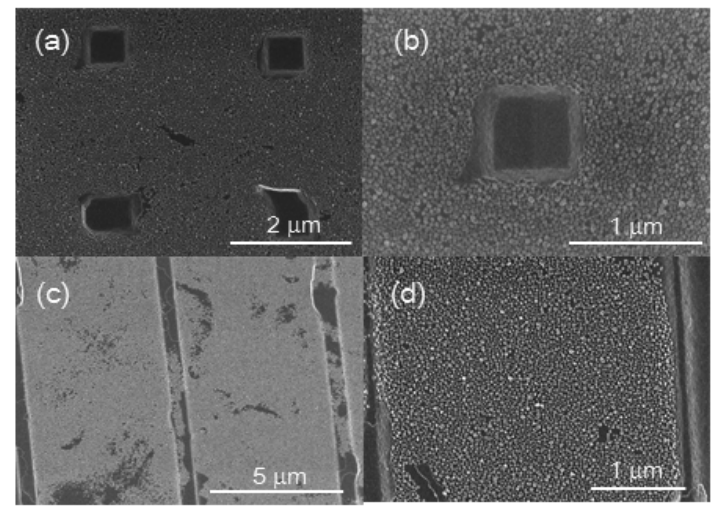

Fig. 5. SEM micrographs of controlled array of $\mathrm{Au}$ nanoparticles supported by dithiol SAMs on $\mathrm{Au}$ patterns; (a) and (b) square patterns, and (c) and (d) line and space patterns.

\section{Conclusion}

We succeeded in controlled array of $\mathrm{Au}$ nanoparticles on nano-patterns using the combination technique of top-down nanofabrication such as nano imprint and bottom-up nanofabrication such as self-assembly. This method enables the controlled array of $\mathrm{Au}$ nanoparticles on the resulting water-soluble dithiol SAM nano-patterns. We can scale up or down the pitch by changing PAK-01 patterns imprinted from quartz glass mold. This method is suitable for self-assembled nanolithography applications in electronics, optics, biosensing, bioimaging and so on.

\section{Acknowledgement}

This work was supported in part by a Grant-in-Aid for Scientific Research (Project No.26706027) from the Ministry of Education, Culture, Sports, Science and Technology of Japan (MEXT). This study was also supported by the Cooperative Research Program of Institute for Catalysis, Hokkaido University (Grant 15A1001). The authors wish to thank Dr. Atsushi Sekiguchi and Ms. Yoko Matsumoto of Litho Tech Japan co. jp for experimental and useful advice as for nano imprint.

\section{References}

1. M. S. Wei, and S. Y. Chou, J. Appl. Phys., 76
(1994) 6679.

2. A. E. Grigorescu, and C. W. Hagen, Nanotechnology, 20 (2009) 29200.

3. H. Schift, J. Vac. Sci. Technol. B26 (2008) 458.

4. S. Y. Chou, P. R. Krauss, and P. J. Renstrom, Appl. Phys. Lett., 67 (1995) 3114.

5. S. Matsui, Y. Kojima, and Y. Ochiai, Appl. Phys. Lett., 53 (1988) 868.

6. H. Masuda and K. Fukuda, Science, 268 (1995) 1466.

7. R. Gupta, J. J. McClelland, Z. J. Jabbour, and R. J. Celotta, Appl. Phys. Lett., 67 (1995) 1378.

8. A. Ulman, An Introduction to Ultrathin Organic Films from Langmuir-Blodgett to Self-Assembly, Academic Press. San Diego, (1991).

9. Y. Xia and G. M. Whitesides, Angew. Chem., 110 (1998) 568.

10. A. D. Kent, T. M. Shaw, S. von Molnar, and D. D. Awschalom, Science, 262 (1993) 1249.

11. X. Liu, L. Fu, S. Hong, V. P. Dravid, and C. A. Mirkin, Adv. Mater., 14 (2002) 231.

12. S. A. Gusev, N. A. Korotkova, D. B. Rozenstein, and A. A. Fraerman, J Appl. Phys., 76 (1994) 1994.

13. A. P. Alivisatos, K. P. Johnsson, X. G. Peng, T. E. Wilson, C. J. Loweth, M. P. Bruchez, and P. G. Schultz, Nature, 382 (1996) 609.

14. M. T. Reetz, M. Winter, G. Dumpich, J. Lohau, and S. Friedrichowski, J. Am. Chem, Soc., 119 (1997) 4539.

15. H. Yamamoto, A. Ohnuma, T. Kozawa and B. Ohtani, J. Photopolym. Sci. Technol., 25 (2012) 449.

16. H. Yamamoto, A. Ohnuma, B. Ohtani and T. Kozawa, Microelectron. Eng., 110 (2013) 369.

17. H. Yamamoto, A. Ohnuma, B. Ohtani and T. Kozawa, J. Photopolym. Sci. Technol., 26 (2013) 495.

18. H. Yamamoto, A. Ohnuma, B. Ohtani and T. Kozawa, Microelectron. Eng., 121 (2014) 108.

19. H. Yamamoto, A. Ohnuma, B. Ohtani and T. Kozawa, J. Photopolym. Sci. Technol., 27 (2014) 243.

20. N. Sakai, J. Taniguchi, K. Kawaguchi, M. Ohtaguchi and T. Hirasawa, J. Photopolymer Sci. Technol., 18 (2005) 531.

21. G. Frens Nature: Phys. Sci. 241 (1973) 20.

22. K. J. Watson, J. Zhu, S. B. T. Nguyen, and C. A. Mirkin, J. Am. Chem. Soc., 121 (1999) 462.

23. A. Ohnuma, R. Abe, T. Shibayama, and B. Ohtani, Chem. Commun., 29 (2007) 3491. 